\title{
Prerequisites for Appropriate Clinical Staging of Major Psychiatric Disorders
}

\author{
Se Hyun Kim $\bowtie$ \\ Department of Neuropsychiatry, Seoul National University Hospital, Seoul, Republic of Korea
}

Clinical staging is useful for describing where an individual presentation lies along the course of illness and the extent of disease progression at a particular timepoint. Staging forms the basis of disease assessment, prognosis, screening, prevention, and determination of appropriate treatment. Clinical staging is widely used in the fields of oncology and cardiology. ${ }^{1,2}$

In the previous issue, Muneer and Mazommi systematically reviewed clinical staging models of major mood disorders and their associated clinical and neurobiological correlates. ${ }^{3}$ Several assumptions must be made to create a useful staging model for a particular disease. First, the natural history of the disorder must be predictable. Second, stage-appropriate treatment must be capable of influencing disease progression. Third, earlier diagnosis and management must afford a favorable prognosis. Last, early intervention can change stage development over time. A great deal of research has allowed us to understand the natural history and pathogenetic mechanisms of many major psychiatric disorders; various novel treatments improve long-term prognoses. Moreover, studies on the premorbid and prodrome stages of psychiatric disorders have shed light on useful preventative approaches. Based on these efforts, the clinical staging of major psychiatric disorders has improved greatly. ${ }^{1,2}$

However, the basis of clinical staging remains of concern. The boundaries of psychiatric disorders shift because of heterogeneity within single diagnostic entities, such that both the clinical course and outcome are unpredictable. Transdiagnostic similarities are also prevalent. As the early stage clinical phenotypes of many mental disorders overlap substantially, lumping rather than splitting during staging has been suggested. ${ }^{4}$ In the bipolar disorder staging model, the phenotypes of chronic disabilities based on the neuroprogression model re-

$\triangle$ Correspondence: Se Hyun Kim, MD, PhD

Department of Neuropsychiatry, Seoul National University Hospital, 101

Daehak-ro, Jongno-gu, Seoul 03080, Republic of Korea

Tel: +82-2-2072-4833, Fax: +82-2-744-7241, E-mail: sh3491@snu.ac.kr

(a) This is an Open Access article distributed under the terms of the Creative Commons Attribution Non-Commercial License (https://creativecommons.org/licenses/by$\mathrm{nc} / 4.0$ ) which permits unrestricted non-commercial use, distribution, and reproduction in any medium, provided the original work is properly cited. lated to allostatic load ${ }^{5,6}$ overlap with those of chronic severe schizophrenia. ${ }^{7.8}$ The chronic refractory states of several psychiatric disorders show similarity in such transdiagnostic features. ${ }^{7.8}$

The categorical diagnostic system has gradually been superseded by the spectrum model of psychiatric disorders. The bipolar spectrum is a widely accepted concept. ${ }^{9}$ The fifth edition of the Diagnostic and Statistical Manual of Mental Disorders (DSM-5) introduced the spectral representation of autism and substance abuse..$^{10}$ The spectrum approach facilitates a dimensional understanding of psychiatric disorders. Single diagnostic entities could be supplanted by a more flexible continuum that considers multiple factors. A staging system provides a heuristic framework for research on psychiatric disorders and appropriate clinical practice; use of an integrated dimensional spectral approach toward psychiatric disorders could assist the construction of appropriate staging systems. $^{11}$

\section{REFERENCES}

1. McGorry PD, Hickie IB, Yung AR, Pantelis C, Jackson HJ. Clinical staging of psychiatric disorders: a heuristic framework for choosing earlier, safer and more effective interventions. Aust N Z J Psychiatry 2006;40:616-622.

2. Berk M, Berk L, Dodd S, Cotton S, Macneil C, Daglas R, et al. Stage managing bipolar disorder. Bipolar Disord 2014:16:471-477.

3. Muneer A, Mazommil R. The staging of major mood disorders: clinical and neurobiological correlates. Psychiatry Investig 2018:15:747-758.

4. Scott J, Leboyer M, Hickie I, Berk M, Kapczinski F, Frank E, et al. Clinical staging in psychiatry: a cross-cutting model of diagnosis with heuristic and practical value. Br J Psychiatry 2013;202:243-245.

5. McEwen BS, Wingfield JC. The concept of allostasis in biology and biomedicine. Horm Behav 2003;43:2-15.

6. Fries GR, Pfaffenseller B, Stertz L, Paz AV, Dargél AA, Kunz M, et al. Staging and neuroprogression in bipolar disorder. Curr Psychiatry Rep 2012;14:667-675.

7. Daban C, Martinez-Aran A, Torrent C, Tabares-Seisdedos R, BalanzaMartinez V, Salazar-Fraile J, et al. Specificity of cognitive deficits in bipolar disorder versus schizophrenia. A systematic review. Psychother Psychosom 2006;75:72-84.

8. Vieta E, Popovic D, Rosa AR, Solé B, Grande I, Frey BN, et al. The clinical implications of cognitive impairment and allostatic load in bipolar disorder. Eur Psychiatry 2013;28:21-29. 
9. Ghaemi SN, Dalley S. The bipolar spectrum: conceptions and misconceptions. Aust N Z J Psychiatry 2014;48:314-324.

10. American Psychiatric Association (2013). DSM-5 Fact Sheets. Available at: https://www.psychiatry.org/psychiatrists/practice/dsm/educa- tional-resources/dsm-5-fact-sheets). Accessed 4 September 2018

11. Guloksuz S, van Os J. The slow death of the concept of schizophrenia and the painful birth of the psychosis spectrum. Psychol Med 2018;48: 229-244. 